\title{
Temperature Field Simulation and Test of Laser Micro-drilling Libin Guo ${ }^{1, a^{*}}$, Yunfei Duan ${ }^{1, b}$, Meng Guo ${ }^{1, c}$, Hai Cui ${ }^{1, d}$, and Fenglong Yao, ${ }^{1, e}$ 1College of Mechanical and Electrical Engineering, Harbin Engineering University, Harbin, 150001, China \\ aguolibin@hrbeu.edu.cn, bdyffeifei@hotmail.com, cguomeng4126188@163.com, ${ }^{d}$ cuihai@hrbeu.edu.cn, ${ }^{\mathrm{e}}$ yaofenglong151@163.com
}

Keywords: Laser, Micro-drilling, Temperature field, Simulation test

Abstract. Temperature field analysis of laser micro-drilling is a nonlinear transient thermal conduction problem with phase changing. In this paper, first the temperature field is simulated with ANSYS finite element analysis software, thus obtaining a three-dimensional temperature field distribution. By analyzing the temperature values of the laser irradiated surfaces, with holes having different diameters, depths and directions, we obtained the law that the temperature varying with time. Finally the temperature variation rate is calculated with the above information. To verify the above simulation and analysis result, laser micro-drilling experiments are conducted. In the tests, the holes' diameter and depth are measured, and their relationship with the measurement laser pulse number is analyzed. Test result shows that the simulation result is correct, thus providing a theoretical evidence for enhancing the dimensional accuracy and surface quality of laser micro-drilling.

\section{Introduction}

With the rapid development of precision and ultra-precision machining technology, laser microprocessing technology is attracting greater attention from research ${ }^{[1]}$. Laser microprocessing at a size of $1 \mu \mathrm{m} \sim 1 \mathrm{~mm}$ parts can be used for drilling, cutting or even three-dimensional machining. Domestic and international researches have conducted the research on the temperature field variation of laser welding, cladding, cutting, and other processing technology, however, there exists less work on the research of the temperature field of micro hole processing ${ }^{[2-4]}$. To do this research, a difficult thermal conduction differential equation needs to be solved first. In this paper, ANSYS software is used for this purpose because it can be used to simulate the temperature field of laser micro-drilling process, to explore the variation of temperature field, and to study the micro hole formation mechanism.

\section{Mathematical Model of Laser Micro-Drilling}

In our experiment, laser beam with approximately Gaussian distribution is used. To simplify the heat transferring analysis for laser processing, the following conditions are assumed ${ }^{[5]}$ :

(1) Materials thermal parameters (coefficient of convective heat transfer $h$ and coefficient of thermal diffusion $\alpha$, etc.) do not vary with temperature.

(2) Melting issue during drilling the material is ignored. Only the gasification issue of the material is considered.

Thus the heat balance equation of two-dimensional nonlinear transient heat transfer in the laser process is established as ${ }^{[6]}$ :

$$
\rho c \frac{\partial T(x, y, t)}{\partial t}=\left[\lambda \frac{\partial^{2} T(x, y, t)}{\partial x^{2}}+\lambda \frac{\partial^{2} T(x, y, t)}{\partial y^{2}}\right],
$$

where, $\rho$ is the density, $c$ is heat capacity, and $\lambda$ is the thermal conductivity.

Suppose $\mathrm{T}$ is environmental temperature, and $\mathrm{T}(\mathrm{x}, \mathrm{y}, 0)=\mathrm{T}_{0}$, where $\mathrm{T}_{0}$ is $25^{\circ} \mathrm{C}$ environmental temperature, thus the boundary conditions are : 
(1) Phase change boundary

$$
\lambda \frac{\partial T(x, y, t)}{\partial z}=-\left[A l(x)-\rho L_{q} v_{r}\right]
$$

where $\lambda$ is thermal conductivity, $\mathrm{A}$ is material absorption coefficient, Lq is latent heat of vaporization, $v_{r}$ is the moving velocity of phase change interface, and $v_{r}=\frac{I_{0}}{\rho\left(L_{q}+a T_{q}\right)}$, where Tq is vaporization temperature. Using Hagen-Rubens formula can calculate the material absorption coefficient $\mathrm{A}$ as :

$$
A(T)_{Y A G}=354.67 \sqrt{a+b T},
$$

where $\mathrm{a}, \mathrm{b}$ are material parameters.

(2) Workpiece periphery boundary

$$
-\lambda \frac{\partial T}{\partial n}=h\left(T-T_{0}\right)+k \mu\left(T^{4}-T_{0}^{4}\right)
$$

where $\frac{\partial T}{\partial n}$ is the rate of temperature change along the workpiece exterior normal, $\mathrm{h}$ is coefficient of convective heat transferring, $\mathrm{k}$ is emission coefficient of the workpiece surface, $\mu$ is blackbody radiation constant.

\section{Temperature Field Simulation of Laser Micro-Drilling}

SUS301 stainless steel is used as workpiece material, which has a higher fatigue resistance strength than the ordinary steel, as well as higher temperature strength and corrosion resistance. Its thermal physical properties are shown in Table 1.

Table 1 SUS301 thermal physical properties

\begin{tabular}{|c|c|c|c|c|c|}
\hline $\begin{array}{c}\text { Temperature } \\
{\left[{ }^{\circ} \mathrm{C}\right]}\end{array}$ & 25 & 1397 & 1430 & 1464 & 1800 \\
\hline $\begin{array}{c}\text { Heat transfer } \\
\text { coefficient } \\
{\left[\mathrm{W} /\left(\mathrm{m} \cdot{ }^{\circ} \mathrm{C}\right)\right]}\end{array}$ & 14 & 33.4 & 32.9 & 28.2 & 30 \\
\hline $\begin{array}{c}\text { Density } \\
{\left[\mathrm{kg} / \mathrm{m}^{3]}\right.}\end{array}$ & 7930 & 7100 & 7000 & 6800 & 6400 \\
\hline
\end{tabular}

The simulation model selects the cylinder model with a radius of $1 \mathrm{~mm}$ with $0.1 \mathrm{~mm}$ thickness; the interior of the workpiece transfers heat using conduction, and the surface has convection. The setting environment temperature is $25^{\circ} \mathrm{C}$.

To simplify the calculation process, the spike pulses are simplified as rectangular pulses, as shown in Fig. 1. Pulse width $t_{1}$ is $0.4 \mathrm{~ms}$, setting a pulse load with power density $\mathrm{q}_{\mathrm{m}}$ in the range $\mathrm{t}_{1}$, the time $t_{1}-t_{2}$ where no pulse load. The time $t_{2}$ corresponds to a complete pulse cycle. 


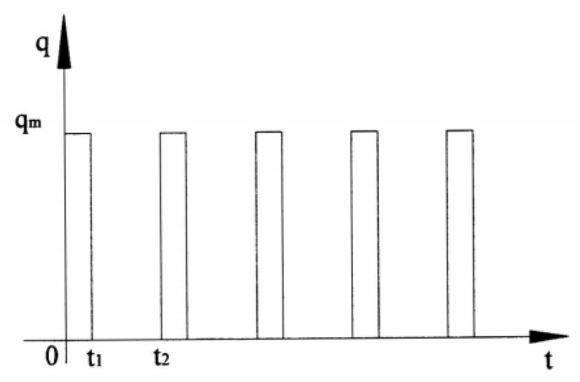

Fig. 1. Pulsed laser energy load varies with time

\section{Simulation results and analysis}

The results from using ANSYS software to simulate SUS301 material laser micro drilling is shown in Fig. 2.

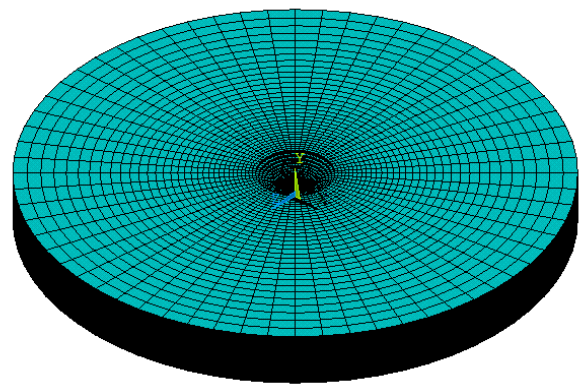

Fig. 2. Laser micro drilling model

In order to show the temperature variation in depth of the micropore in the hole depth direction more clearly, the model is cut into quarters. The observed change in temperature in the hole depth direction is shown in Fig. 3.

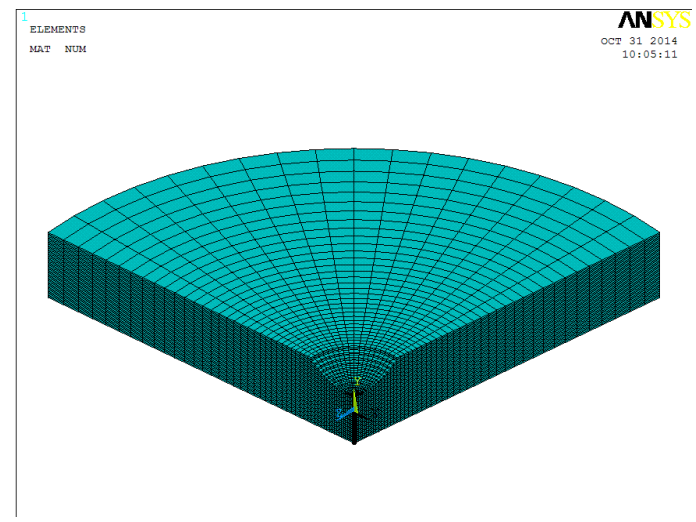

Fig. 3. Laser micro drilling model in the hole depth direction

The model is loaded by the post-processing module of ANSYS software, and then the obtained results are imported into the processor. When the pulse energy is $75.4 \mathrm{~mJ}$, the irradiation surface and temperature field distribution of the single pulse laser micro drilling in the hole depth direction are shown in Fig. 4 and Fig. 5. 


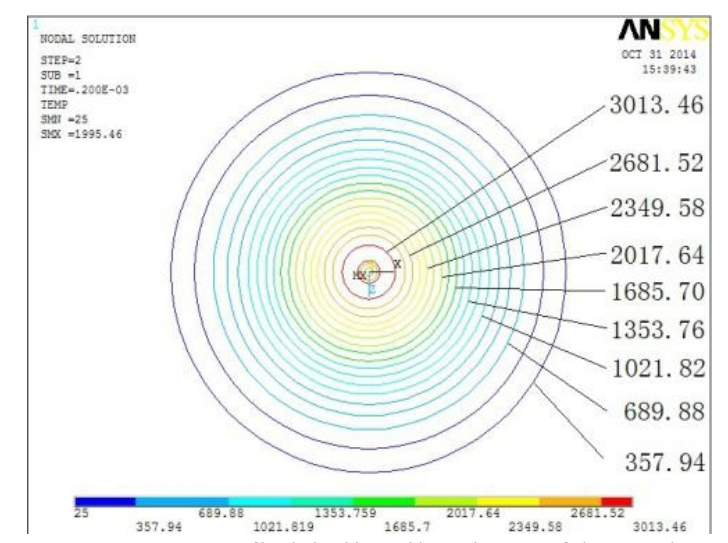

Fig. 4. Temperature field distribution of laser beam spot

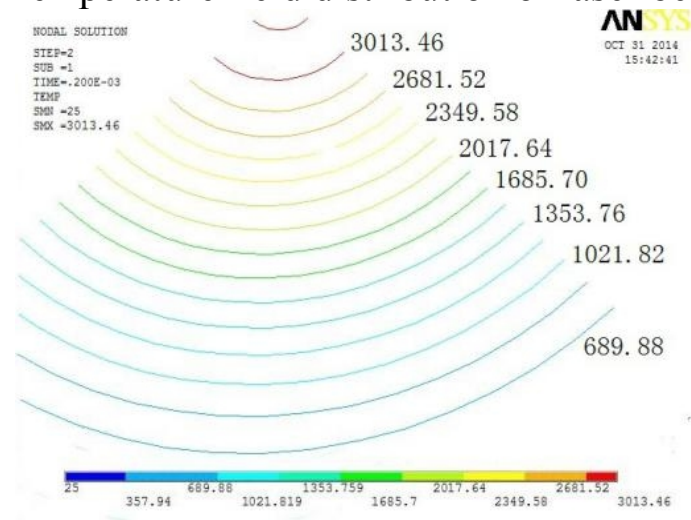

Fig. 5. Temperature field distribution in the hole depth direction

In Fig. 4 , the software calculates that from $0.1 \sim 0.3 \mathrm{~ms}$ the material central temperature is up to $3013^{\circ} \mathrm{C}$. With the increase of the spot radius $\mathrm{r}$, the temperature decreases. When the temperature decreases from $3013{ }^{\circ} \mathrm{C}(\mathrm{r} \approx 0 \mu \mathrm{m})$ to $1353{ }^{\circ} \mathrm{C}$, the spot radius $\mathrm{r} \approx 60 \mu \mathrm{m}$. Fig. 5 shows that the energy is gradually transferred to the interior of the workpiece. With the increase in depth, the temperature is gradually reduced and the rate of decrease on the surface is faster than the surface irradiated by the laser spot. When the temperature was lowered from $3013{ }^{\circ} \mathrm{C}$ to $1353{ }^{\circ} \mathrm{C}$, the depth is $30 \mu \mathrm{m}$. Due to the melting point of steel being $1371 \sim 1398^{\circ} \mathrm{C}$, if without considering other factors, this pulse laser can process a micropore with the diameter $120 \mu \mathrm{m}$ and $30 \mu \mathrm{m}$ depth. Because the laser energy in space follows a Gaussian distribution, the major part of the energy is usually concentrated near the center of the spot and the maximum intensity of the light field outside the central area is not more than $2 \%$ of the maximum intensity of the entire light field. Therefore, the rate of the temperature decline outside the central region is linear, as shown in Fig. 6.

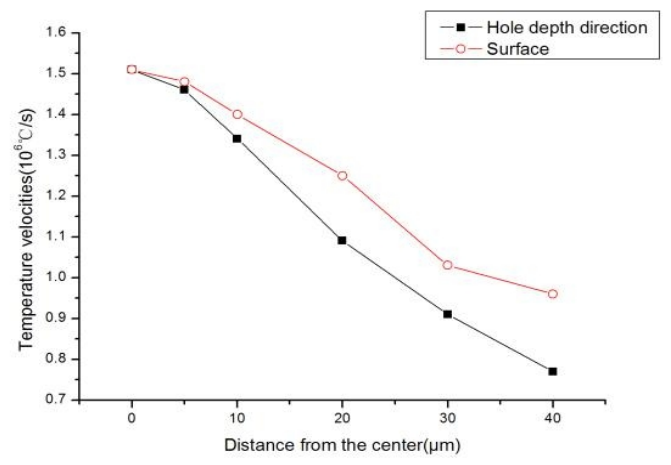

Fig. 6. The irradiated surface and hole depth direction temperature change rate curve at different position 


\section{Single pulse and multi-pulse tests and analysis}

The experiments use JHM-1GY-120 type precise laser machine with a wavelength of $1.06 \mu \mathrm{m}$, pulse width of $0.4 \mathrm{~ms}$, and pulse energy of $75.4 \mathrm{~mJ}$. The single pulse and multi-pulse micro drilling experiments are conducted using SUS301 stainless steel as processing materials. The diameter and the hole depth are measured with a digital microscope as shown in Fig. 7.

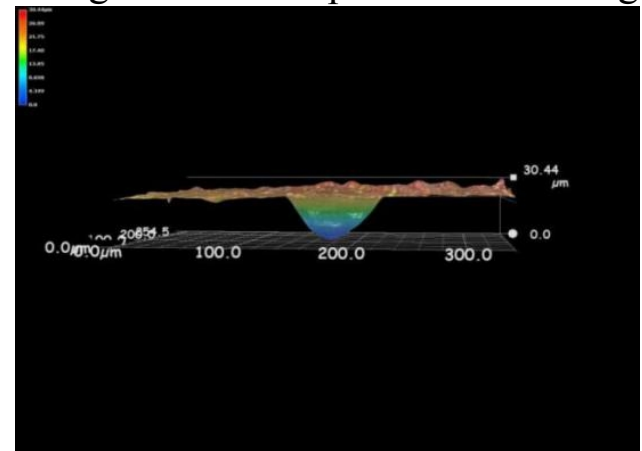

Fig. 7. Three dimensional micro hole structure using digital microscope

The curves of the diameter and hole depth variation with the number of pulses is obtained using the Origin software, which analyzes simulation and measurement results, as shown in Figs. 8 and 9.

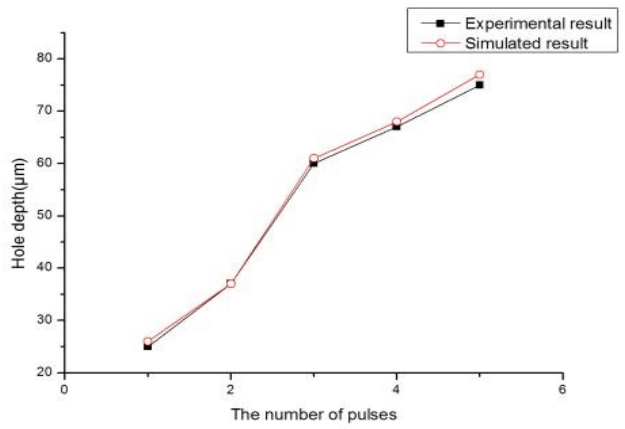

Fig. 8. Curves of hole depth variation with the number of pulses

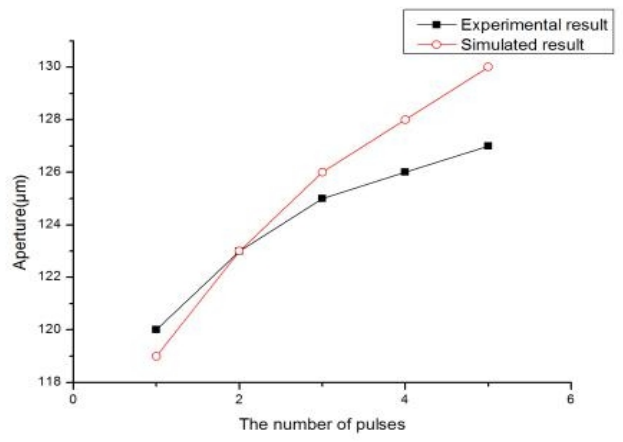

Fig. 9. Curves of the hole diameter variation with the number of pulses

Though the trends of the simulation value and the measured value of the hole diameter are the same, the calculated values are greater than the measured ones. Especially in the third pulse, the differences between the simulated and measured values are gradually increasing. This is because in this stage, as the hole depth increases, the incident angle of the laser is reduced. As a result, the laser in the hole wall leaves energy internally reflected to make the aggregation move down, so little changes are measured in diameter values as shown in Fig. 10 ${ }^{[7]}$. 


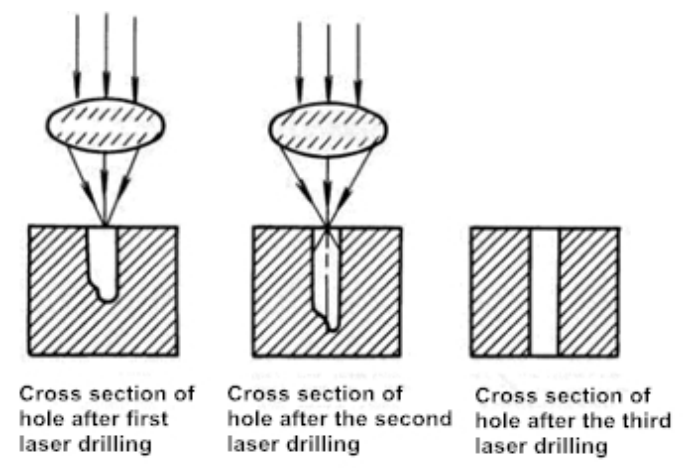

Fig. 10. Diagram of lights reaching the hole wall ${ }^{[7]}$

The comparison shows that the simulation accuracy is higher, which can provide the basis for process parameter selection of laser micro drilling.

\section{Conclusions}

From the research of temperature field simulation of the laser micro-drilling process, we reach the following conclusions:

(1) It establishes a thermodynamic mathematical model of the laser micro drilling. Through studying the temperature field distribution simulation of the surface irradiated by the laser and hole depth direction, the results show that the spot center temperature reaches $3013{ }^{\circ} \mathrm{C}$ within $0.4 \mathrm{~ms}$, which is much higher than the melting point and boiling point of stainless steel.

(2) The rate of temperature change on the laser irradiation surface and the hole depth direction increases with the radius and the depth, with the maximum difference being up to $105^{\circ} \mathrm{C} / \mathrm{s}$. This causes the large dimension differences between the hole size and the depth, forming conical shape holes. Therefore, machining the straight wall micro holes requires multi-pulse processing.

(3) The laser micro-drilling experiment analyzes the relationships of hole depth, hole size and number of pulses and verifies the accuracy of the simulation method. This can provide the theoretical basis for improving the machining accuracy, productivity and surface quality of laser micro drilling.

\section{References}

[1] Kai Peng, Yuhang Peng and Hongzhen Hou, Present situation and prospects of laser processing technology development, J. Shandong Industrial Technology. 20 (2014) 39-40. In Chinese

[2] E. Toyserkani, A. Khajepour and S. Corbin, 3-D finite element modeling of laser cladding by power injection: effects of laser pulse shaping on the process, J. Optics and Laser in Engineer. 41 (2004) 849-867.

[3] A. Ancona, F. Röser, K. Rademaker, J. Limpert, High speed laser drilling of metals using a high repetition rate, high average power ultrafast fiber CPA system, J. Optics Express. 16 (2008) 8958-8968.

[4] Hao Liu, Gang Yu, Xiuli He, et al, Three-dimensional numerical simulation of transient temperature field and coating gemometry in power feeding laser cladding, J. Chinese Journal of Lasers. 40(2013): 1203007. In Chinese

[5] Jinqi Zhang, Jianmei Li, Changhou Lu, Xinxin Fang, Zhuo Ma, Study on the numerical simulation technology of laser thermal effect, J. Journal of Machanical Electrical Engineering. 11 (2014) 1371-1378. In Chinese

[6] Jiale $\mathrm{Xu}$, Zhongguo Li, Hhuafeng Guo, Xizhi Jiang, Kai Wang, Numerical simulation of the temperature field and tribological properties of AZ91D magnesium alloy with laser remelting, J. Journal of Materials Protecion. 3 (2014) 8-11. In Chinese

[7] Jicheng Bai, Yongfeng Guo, Jinchun Liu, Technology of non-traditional machining, Harbin Institute of Technology press, Harbin, 2006, pp. 95. 